\title{
Evaluation of the genotoxic and mutagenic potentials of a living nosode compounded with Influenza A virus
}

\author{
Juliana Patrão de Paiva, Camila Monteiro Siqueira, Carla Holandino, \\ Alvaro da Costa Leitão
}

Federal University of Rio de Janeiro, Rio de Janeiro, Brazil

\begin{abstract}
Background: The influenza virus has been responsible for contagious respiratory diseases with high mortality rates [1]. Some drugs have been used to treat human influenza. However, these drugs cause many common side effects and induce the appearance of resistant viral strains [2]. The impact caused by the influenza virus has motivated the development of new approaches for the prevention and control of influenza [3]. Therefore, a new homeopathic medicine was developed using, as a starting point, the infectious influenza virus [4]. This belongs to a group called living nosodes [5]. However, its mutagenic and genotoxic potentials, especially when used in low dilutions, has not yet been evaluated and it is important because this biotherapic is prepared from living microorganisms. Different methods can be used to detect mutagenic and genotoxicic effects.
\end{abstract}

Aims: This study aims to evaluate the genotoxic and mutagenic potentials of influenza A living nosode at different homeopathic potencies.

Methodology: $1 \mathrm{ml}$ of purified viral suspension was diluted in $9 \mathrm{ml}$ of sterile distilled water. This sample was submitted to 100 mechanical succussions (approximately $3 \mathrm{~Hz}$ ), using Autic ${ }^{\circledR}$ Brazilian machine, originating the first dilution, named decimal (1x). $1 \mathrm{ml}$ of this solution was diluted in $9 \mathrm{ml}$ of solvent and was submitted to 100 sucussions, generating biotherapic $2 x$. This procedure was successively repeated, according to Brazilian Homeopathic Pharmacopoeia, to obtain the biotherapic 30x [6]. By the same technique, water vehicle was prepared until 30x potency to be used as control. All samples were prepared in sterile and under aseptic conditions, using laminar flow cabinet, class II, and were stored in the refrigerator $\left(8^{\circ} \mathrm{C}\right)$. The samples $1 \mathrm{x}, 6 \mathrm{x}$, $12 \mathrm{x}, 18 \mathrm{x}, 24 \mathrm{x}$ and 30x and water 30x (vehicle control) were analysed by: the Inductest, which assesses the ability of physical or chemical agents to promote lysogenic induction as a reflection of damage in DNA molecules in lysogenic bacteria, and the Ames test, which uses indicator strains of Salmonella typhimurium, sensitive to substances that can induce different types of mutation.

Results: The Inductest showed no decrease in the survival fraction of the bacteria used, and no increase in the formation of lysogenic induction, in any tested potency. The same profile was obtained after the Ames test, with similar results to negative control.

Conclusion: We can conclude that this living nosode compounded with Influenza A virus is not able to induce DNA damage in prokaryotic cells. This result permits us to conclude that patients who use this medicine have no side effects related to mutagenesis and genotoxicity.

Key words: Living nosode; Biotherapic; Homeopathy; Genotoxicity; Mutagenesis. 


\section{References:}

[1] Couceiro JNSS, Couceiro ESS, Pinto AMV, Machado RD, Cabral MC. Comparative evaluation of a simple and sensitive assay for detection of orthomyxo and paramyxoviruses. Memórias do Instituto Oswaldo Cruz. 1994; 89(2):183-187.

[2] Monto AS, McKimm-Breschkin JL, Macken C, Hampson AW, Hay A, Klimov A, Tashiro M, Webster RG, Aymard M, Hayden FG, Zambon M. Detection of influenza viruses resistant to neuraminidase inhibitors in global surveillance during the first 3 years of their use. Antimicrobial Agents and Chemotherapy. 2006; 50(7):2395-2402.

[3] Forleo-Neto E, Halker E, Santos VJ, Paiva TM, Toniolo-Neto J. Influenza. Revista da Sociedade Brasileira de Medicina Tropical. 2003; 36(2):267-274.

[4] Siqueira CM. Cellular changes induced by a new live biotherapic-type nosode in the MDCK and J774.G8 lines [dissertation]. Rio de Janeiro (Brazil): College of Pharmacy, Federal University of Rio de Janeiro; 2009. $128 \mathrm{p}$.

[5] Costa, RA. Nosódios vivos. 1st ed. Rio de Janeiro: Farmacia Homeopatica Atomo LTDA; 2002.

[6] Brazilian Homeopathic Pharmacopoeia, 2nd ed. São Paulo: Atheneu; 1997.

\section{Avaliação do potencial genotóxico e mutagênico de um nosódio vivo obtido com o vírus Influenza A}

\section{RESUMO}

Introdução: $\mathrm{O}$ vírus da gripe tem sido responsável por doenças respiratórias contagiosas com altas taxas de mortalidade [1]. Algumas drogas tem sido usadas para tratar a gripe humana. No entanto, esses medicamentos causam muitos efeitos colaterais e induzem o aparecimento de cepas virais resistentes [2]. $\mathrm{O}$ impacto causado pelo vírus influenza tem motivado o desenvolvimento de novas abordagens para a prevenção e controle da influenza [3]. Portanto, um novo medicamento homeopático foi desenvolvido utilizando, como ponto de partida, o vírus influenza infecciosa [4]. Este pertence a um grupo chamado nosódios vivos [5]. No entanto, seus potenciais mutagênicos e genotóxicos, especialmente quando usado em diluições baixas, ainda não foram avaliados e é importante porque este bioterápico é preparado a partir de microorganismos vivos. Diferentes métodos podem ser usados para detectar efeitos mutagênicos e genotóxicos.

Objetivos: Este estudo visa avaliar o potencial genotóxico e mutagênico do nosódio vivo do vírus influenza A, em diferentes potências homeopáticas.

Metodologia: $1 \mathrm{ml}$ de suspensão viral purificada foi diluída em $9 \mathrm{ml}$ de água destilada estéril. Esta amostra foi submetida a 100 sucussões mecânicas (aproximadamente $3 \mathrm{~Hz}$ ), usando o aparato Autic ${ }^{\circledR}$ brasileira, originando a primeira diluição, chamada decimal (1x). $1 \mathrm{ml}$ desta solução foi diluída em $9 \mathrm{ml}$ de solvente e foi submetido a 100 sucussões, gerando o bioterápico 2x. Este procedimento foi repetido sucessivamente, de acordo com a Farmacopéia Homeopática Brasileira, para obter o bioterápico 30x [6]. Pela mesma técnica, a água foi preparada até a potência 30x, para ser utilizada como controle. Todas as amostras foram preparadas 
sob condições estéreis e assépticas, utilizando-se fluxo laminar, classe II, e foram armazenados em geladeira $\left(8^{\circ} \mathrm{C}\right)$. As amostras 1x, 6x, 12x, 18x 24x, 30x e 30x e água (controle do veículo) foram analisadas por: Induteste, avalia a capacidade dos agentes físicos ou químicos de promover a indução lisogênico como um reflexo dos danos nas moléculas de DNA em bactérias lisogênicas, e o teste de Ames, que utiliza linhagens indicadoras de Salmonella typhimurium, sensíveis a substâncias que podem induzir diferentes tipos de mutação.

Resultados: Os resultados obtidos no Induteste não mostraram diminuição da fração de sobrevivência das bactérias utilizadas, e nenhum aumento na formação de indução lisogênica, em quaisquer potências testadas. O mesmo perfil foi obtido após o teste de Ames, com resultados semelhantes ao controle negativo.

Conclusão: Conclui-se que nosódio vivo obtido com vírus Influenza A não é capaz de induzir danos no DNA de células procarióticas. Este resultado nos permite concluir que pacientes que usam este medicamento não tem efeitos colaterais relacionados com a mutagênese e genotoxicidade.

Palavras-chave: Nosódio vivo; Bioterápico, Homeopatia; Genotoxicidade; Mutagênese.

\section{(c) EY-NC-ND Licensed to GIRI}

Support: IBCCF/UFRJ, FF/UFRJ, FAPERJ, CNPq; FUJB, CAPES

Conflict of interest: authors declare there is no conflict of interest

Correspondence author: Juliana Patrão de Paiva, jupaiva_rj@hotmail.com. Multidisciplinary Laboratory of Pharmaceutical Sciences, Federal University of Rio de Janeiro, School of Pharmacy, Center for Health Sciences, CCS, Block B - basement, Rio de Janeiro - RJ - Brazil - Phone (21) 2280-1784

How to cite this article: Paiva JP, Siqueira CM, Holandino C, Leitão AC. Evaluation of the genotoxic and mutagenic potentials of a living nosode compounded with Influenza A virus. Int J High Dilution Res [online]. 2011 [cited YYYY Month dd]; 10(36): 180-182. Proceedings of the XXV GIRI Symposium and VIII CBFH; 2011 Sep 04-07; Foz do Iguaçu (Brazil). GIRI and ABFH; 2011; Available from: http://www.feg.unesp.br/ ojs/index.php/ijhdr/article/view/502/512 